\title{
CONVOLUTION EQUATIONS IN SPACES OF DISTRIBUTIONS WITH ONE-SIDED BOUNDED SUPPORT \\ BY
}

R. SHAMBAYATI AND Z. ZIELEZNY

\begin{abstract}
Let $\mathscr{D}^{\prime}(0, \infty)$ be the space of distributions on $R$ with support in $[0, \infty)$ and $\mathscr{S}^{\prime}(0, \infty)$ its subspace consisting of tempered distributions. We characterize the distributions $S \in \mathscr{D}^{\prime}(0, \infty)$ for which $S * \mathscr{D}^{\prime}(0, \infty)=\mathscr{D}^{\prime}(0, \infty)$, where * is the convolution. We also characterize the distributions $S \in \mathscr{S}^{\prime}(0, \infty)$ for which $S * \mathscr{S}^{\prime}(0, \infty)=\mathscr{S}^{\prime}(0, \infty)$.
\end{abstract}

The problem of existence of solutions of convolution equations in spaces of distributions on $R^{n}$ was thoroughly investigated by L. Ehrenpreis [1]. Later, L. Hörmander [2] gave a systematic exposition of the range of convolution operators in spaces of distributions on arbitrary open sets $\Omega \subset R^{n}$. In particular, consider the space $\mathscr{D}^{\prime}$ of all distributions on $R^{n}$ and its subspace $\mathscr{E}^{\prime}$ consisting of distributions of compact support. If $S \in \mathscr{E}^{\prime}$, the convolution equation

$$
S * u=v
$$

has a solution $u \in \mathscr{D}^{\prime}$, for every $v \in \mathscr{D}^{\prime}$, if and only if the Fourier transform $\hat{S}$ of $S$ has the following property:

There are constants $A_{1}, A_{2}$ and $A_{3}$ such that for every $\xi \in R^{n}$ there exists $\eta \in R^{n}$ satisfying the conditions

$$
|\xi-\eta| \leqslant A_{1} \log (2+|\xi|) \text { and }|\hat{S}(\eta)| \geqslant\left(A_{2}+|\xi|\right)^{-A_{3}}
$$

In that case $S$ is said to be invertible and $\hat{S}$ slowly decreasing.

However, if we consider the space $\mathscr{D}^{\prime}(0, \infty)$ of distributions of one variable with support in $[0, \infty)$, then the convolution $S * u$ is defined for any $S, u \in \mathscr{D}^{\prime}(0, \infty)$ as a distribution in $\mathscr{D}^{\prime}(0, \infty)$. The question now arises: Under what conditions on $S \in \mathscr{D}^{\prime}(0, \infty)$ has equation (1) a solution $u \in \mathscr{D}^{\prime}(0, \infty)$ for every $v \in \mathscr{D}^{\prime}(0, \infty)$ ? Since the Fourier transform $\hat{S}$ of a distribution $\hat{S} \in \mathscr{D}^{\prime}(0, \infty)$ is, in general, not even a distribution, conditions (2) are now meaningless.

In this paper we characterize the distributions $S \in \mathscr{D}^{\prime}(0, \infty)$ for which $S * \mathscr{D}^{\prime}(0, \infty)=\mathscr{D}^{\prime}(0, \infty)$. We also solve the problem of existence of solutions of equation (1) in the space $\mathscr{S}^{\prime}(0, \infty)$ of tempered distributions with support in $[0, \infty)$.

Received by the editors July 6, 1984.

1980 Mathematics Subject Classification. Primary 45E10; Secondary 46F12.

(C)1985 American Mathematical Society $0002-9947 / 85 \$ 1.00+\$ .25$ per page 
1. Preliminaries. We denote by $\mathscr{D}_{-}$the space of all $C^{\infty}$-functions with support bounded from the right and by $\mathscr{D}_{+}^{\prime}$ its dual space. $\mathscr{D}_{+}^{\prime}$ is the space of distributions with support bounded from the left. We also denote by $\mathscr{D}^{\prime}(\alpha, \infty)$ the space of distributions with support in $[\alpha, \infty)$. For details about these spaces we refer to [4].

If $S, T \in \mathscr{D}_{+}^{\prime}$, then the convolution $S * T$ is well defined as a distribution in $\mathscr{D}_{+}^{\prime}$. We recall some of the properties of the convolution which we apply later. First we have

$$
\operatorname{supp}(S * T) \subset \operatorname{supp} S+\operatorname{supp} T .
$$

In particular, if $S \in \mathscr{D}^{\prime}(\alpha, \infty)$ and $T \in \mathscr{D}^{\prime}(\beta, \infty)$, then $S * T \in \mathscr{D}^{\prime}(\alpha+\beta, \infty)$.

Furthermore, the following theorem of Titchmarsh (see [3 and 6]) is valid for distributions in $\mathscr{D}^{\prime}(0, \infty)$ :

If $S$ and $T$ are distributions in $\mathscr{D}^{\prime}(0, \infty)$ and $S * T \in \mathscr{D}^{\prime}(\gamma, \infty), \gamma>0$, then $S \in \mathscr{D}^{\prime}(\alpha, \infty)$ and $T \in \mathscr{D}^{\prime}(\beta, \infty)$, where $\alpha+\beta=\gamma, \alpha, \beta \geqslant 0$.

Finally, if $S \in \mathscr{E}^{\prime}$, then the value of the convolution $S * T$ on an open set $\Omega$ depends only on the value of $T$ in $\Omega-\operatorname{supp} S$.

We denote by $\mathscr{S}^{\prime}(0, \infty)$ the space of tempered distributions with support in $[0, \infty)$. Distributions in $\mathscr{S}^{\prime}(0, \infty)$ can be characterized in terms of their Fourier transforms (see [5]). Let $C_{-}=\{\zeta \in C: \operatorname{Im} \zeta<0\}$ and denote by $M_{n}$ the function defined on $(-\infty, 0)$ by

$$
M_{n}(\eta)= \begin{cases}1 & \text { if } \eta \leqslant-1, \\ |\eta|^{-n} & \text { if }-1<\eta<0\end{cases}
$$

here $n$ is an integer. A tempered distribution $T$ is in $\mathscr{S}^{\prime}(0, \infty)$ if and only if its Fourier transform $\hat{T}$ can be continued in $C_{-}$to an analytic function $\hat{t}$ satisfying the estimate

$$
|\hat{t}(\zeta)| \leqslant C(1+|\zeta|)^{m} M_{n}(\eta),
$$

where $\zeta=\xi+i \eta, m$ and $n$ are positive integers and $C$ is a constant.

If $S$ and $T$ are both in $\mathscr{S}^{\prime}(0, \infty)$ and if $\hat{s}$ and $\hat{t}$ are the corresponding analytic continuations in $C_{-}$of their Fourier transforms, then $\hat{s} \hat{t}$ is the analytic continuation of a tempered distribution $\hat{S} \circ \hat{T}$ such that

$$
\widehat{S * T}=\hat{S} \circ \hat{T} \text {. }
$$

2. The case of convolution operators of compact support. We first consider convolution operators $S$ with compact support and assume that $\operatorname{supp} S \subset[0, \infty)$; in that case $S * \mathscr{D}^{\prime}(0, \infty) \subset \mathscr{D}^{\prime}(0, \infty)$.

If $S * \mathscr{D}^{\prime}(0, \infty)=\mathscr{D}^{\prime}(0, \infty)$, then $0 \in \operatorname{sing} \operatorname{supp} S$. In fact, if sing supp $S \subset[\alpha, \infty)$, $\alpha>0$, then sing supp $S * u \subset[\alpha, \infty)$ for every $u \in \mathscr{D}^{\prime}(0, \infty)$, since the value of $S * u$ in $(-\infty, \alpha)$ depends only on the value of $S$ in $(-\infty, \alpha)$. This shows that $S * \mathscr{D}^{\prime}(0, \infty) \neq \mathscr{D}^{\prime}(0, \infty)$.

In what follows we use the notion of invertibility of a convolution operator $S \in \mathscr{E}^{\prime}$ in the same sense as in [1] (see the introduction). We also denote by $R_{j}^{-}$and $R_{j}^{+}, j=0, \pm 1, \pm 2, \ldots$, the intervals $(-\infty, j]$ and $[j, \infty)$, respectively. 
TheOREM 1. If $S * \mathscr{D}^{\prime}(0, \infty)=\mathscr{D}^{\prime}(0, \infty)$, then $S$ is invertible and satisfies the conditions

(6)

$u \in \mathscr{D}_{+}^{\prime}, \quad \operatorname{sing} \operatorname{supp}(S * u) \subset R_{j}^{+} \Rightarrow \operatorname{sing} \operatorname{supp} u \subset R_{j}^{+}, \quad j=0, \pm 1, \pm 2, \ldots$

Proof. If $\varphi \in \mathscr{D}$, we pick $h \geqslant 0$ such that supp $\tau_{h} \varphi \subset[0, \infty)$. By assumption, there exists $u \in \mathscr{D}^{\prime}(0, \infty)$ such that $S * u=\tau_{h} \varphi$. Hence $S * \tau_{-h} u=\varphi$, and, obviously, $\tau_{-h} u \in \mathscr{D}^{\prime}$. In other words, we proved that $S * \mathscr{D}^{\prime} \supset \mathscr{D}$, which implies that $S$ is invertible by Theorem 2.5 in [1].

Furthermore, it follows from the hypothesis that $S$ has a fundamental solution in $\mathscr{D}^{\prime}(0, \infty)$, i.e. there exists $E \in \mathscr{D}^{\prime}(0, \infty)$ such that $S * E=\delta$, where $\delta$ is the Dirac measure. Consequently, every distribution $u \in \mathscr{D}^{\prime}(0, \infty)$ can be represented in the form $u=(S * u) * E$. If now $\operatorname{sing} \operatorname{supp}(S * u) \subset R_{j}^{+}$, then $S * u$ is a $C^{\infty}$-function on the complement of $R_{j}^{+}$, and therefore $u=(S * u) * E$ is a $C^{\infty}$-function on $(-\infty, j)$. Thus sing supp $u \subset R_{j}^{+}$.

REMARK 1. If $S$ is any distribution in $\mathscr{D}^{\prime}(0, \infty)$ such that $0 \in \operatorname{supp} S$, then it follows from the Titcinmarsh theorem that

$$
u \in \mathscr{D}_{+}^{\prime}, \quad \operatorname{supp}(S * u) \subset R_{j}^{+} \Rightarrow \operatorname{supp} u \subset R_{j}^{+},
$$

$j=0, \pm 1, \pm 2, \ldots$. In fact, pick an integer $k \geqslant 0$ such that $\tau_{k} u \in \mathscr{D}^{\prime}(0, \infty)$. If $\operatorname{supp}(S * u) \subset R_{j}^{+}$, we have $\operatorname{supp}\left(S * \tau_{k} u\right)=\operatorname{supp} \tau_{k}(S * u) \subset R_{j+k}^{+}$. But $0 \in \operatorname{supp} S$, and so supp $\tau_{k} u \subset R_{j+k}^{+}$, by Titchmarsh's theorem. Hence supp $u \subset R_{j}^{+}$.

REMARK 2. Conditions (6) and (7) are equivalent to the conditions

$$
\begin{gathered}
u \in \mathscr{D}_{-}^{\prime}, \quad \operatorname{sing} \operatorname{supp}(\check{S} * u) \subset R_{j}^{-} \Rightarrow \operatorname{sing} \operatorname{supp} u \subset R_{j}^{-}, \\
u \in \mathscr{D}_{-}^{\prime}, \quad \operatorname{supp}(\check{S} * u) \subset R_{j}^{-} \Rightarrow \operatorname{supp} u \subset R_{j}^{-},
\end{gathered}
$$

$j=0, \pm 1, \pm 2, \ldots$, where $\check{S}$ is the symmetric distribution to $S$. This follows im-

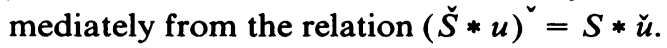

We now prove the converse of Theorem 1 .

TheOREM 2. If $S$ is invertible and satisfies conditions (6), then $S * \mathscr{D}^{\prime}(0, \infty)=$ $\mathscr{D}^{\prime}(0, \infty)$.

Proof. It suffices to show that

$$
S * \mathscr{D}_{+}^{\prime}=\mathscr{D}_{+}^{\prime}
$$

and then apply Titchmarsh's theorem. The proof of (10) is basically the same as that of Theorem 4.5 in [2]. We therefore restrict ourselves to a brief sketch of the proof.

Let $v$ be an arbitrary distribution in $\mathscr{D}_{+}^{\prime}$ and let $p$ be a seminorm in $\mathscr{D}_{-}$such that

$$
|v(\varphi)| \leqslant p(\varphi), \quad \varphi \in \mathscr{D}_{-} .
$$

The main part of the proof is the construction of a seminorm $q$ in $\mathscr{D}_{-}$satisfying the condition

$$
p(\varphi) \leqslant q(\check{S} * \varphi), \quad \varphi \in \mathscr{D}_{-} .
$$


If this is accomplished, conditions (11) and (12) yield $|v(\varphi)| \leqslant q(\check{S} * \varphi), \varphi \in \mathscr{D}_{-}$, and hence, by the Hahn-Banach theorem, the linear form $\check{S} * \varphi \rightarrow v(\varphi), \varphi \in \mathscr{D}_{-}$, can be extended to a linear form $u$ on $\mathscr{D}_{-}$such that $|u(\psi)| \leqslant q(\psi), \psi \in \mathscr{D}_{-}$. Thus $u \in \mathscr{D}_{+}^{\prime}$, and since $u(\check{S} * \varphi)=v(\varphi), \varphi \in \mathscr{D}_{-}$, we have $S * u(\varphi)=v(\varphi), \varphi \in \mathscr{D}_{-}$, or $S * u=v$.

The construction of $q$ is based on the following lemma.

LEMMA. Let $q$ be a seminorm in $\mathscr{D}_{-}$such that

$$
q(\psi) \geqslant \sup _{\alpha \leqslant x}|\psi(x)|, \quad \psi \in \mathscr{D}_{-},
$$

for some $\alpha \in R$, and assume that

$$
p(\varphi) \leqslant q(\check{S} * \varphi), \quad \varphi \in \mathscr{D}_{-}, \operatorname{supp} \varphi \subset R_{j}^{-},
$$

where $j>\alpha$. Then, under the conditions of the theorem, for every $\varepsilon>0$ there exists another seminorm $q^{\prime}$ in $\mathscr{D}_{-}$such that

$$
p(\varphi) \leqslant q^{\prime}(\check{S} * \varphi), \quad \varphi \in \mathscr{D}_{-}, \operatorname{supp} \varphi \subset R_{j+1}^{-},
$$

and

$$
q^{\prime}(\psi)=(1+\varepsilon) q(\psi), \quad \psi \in \mathscr{D}_{-}, \operatorname{supp} \psi \subset R_{j-1}^{-}
$$

We observe that $q(\psi)$ depends only on the values of $\psi$ in $R_{\lambda}^{+}$for some $\lambda \in R$. Otherwise one could find a sequence of functions $\psi_{n} \in \mathscr{D}_{-}$such that supp $\psi_{n} \subset R_{\lambda_{n}}^{-}$, where $\lambda_{n} \rightarrow-\infty$, and $q\left(\psi_{n}\right) \geqslant 1$. Since $\psi_{n} \rightarrow 0$ in $\mathscr{D}_{-}$, this would be a contradiction.

In view of that, the conditions of the lemma need only to be satisfied by functions with compact support and the proof is then the same as in [2].

Given $\varepsilon_{j}>0$ such that $\sum_{j=1}^{\infty} \varepsilon_{j}<\infty$, one can now apply the lemma to successively construct seminorms $q_{j}$ in $\mathscr{D}_{-}$such that

$$
q_{j+1}(\psi)=\left(1+\varepsilon_{j}\right) q_{j}(\psi), \quad \psi \in \mathscr{D}_{-}, \operatorname{supp} \psi \subset R_{j-1}^{-},
$$

and

$$
p(\varphi) \leqslant q_{j}(\check{S} * \varphi), \quad \varphi \in \mathscr{D}_{-}, \operatorname{supp} \varphi \subset R_{j+1}^{-} .
$$

If $q(\psi)=\lim _{j \rightarrow \infty} q_{j}(\psi)$, then $q$ is a continuous seminorm in $\mathscr{D}_{-}$and conditions (13) imply (12).

COROllary 1. For $S \in \mathscr{E}^{\prime}$ with $\operatorname{supp} S \subset[0, \infty)$, the following conditions are equivalent.

(i $) S * \mathscr{D}^{\prime}(0, \infty)=\mathscr{D}^{\prime}(0, \infty)$.

$\left(\mathrm{i}_{2}\right) S * \mathscr{D}_{+}^{\prime}=\mathscr{D}_{+}^{\prime}$.

$\left(\mathrm{i}_{3}\right)$ There exists a fundamental solution for $S$ in $\mathscr{D}^{\prime}(0, \infty)$.

$\left(\mathrm{i}_{4}\right) S$ is invertible and satisfies conditions (6).

3. The general case. We now consider equation (1), where $S$ is an arbitrary distribution in $\mathscr{D}^{\prime}(0, \infty)$. We prove that the solvability of this equation in $\mathscr{D}^{\prime}(0, \infty)$ depends only on the values of $S$ in an arbitrary small neighborhood of the origin. 
TheOREM 3. If $S \in \mathscr{D}^{\prime}(0, \infty)$ and $S * \mathscr{D}^{\prime}(0, \infty)=\mathscr{D}^{\prime}(0, \infty)$, then, for every $\alpha>0, S$ admits a decomposition

$$
S=S_{1}+S_{2}
$$

where $S_{1}$ is a distribution in $\mathscr{E}^{\prime}$ satisfying the equivalent conditions in Corollary 1 , and $S_{2} \in \mathscr{D}^{\prime}(\alpha, \infty)$.

Proof. Given $\alpha>0$, we can always decompose $S$ as in (14), with $S_{1} \in \mathscr{E}^{\prime}$ and $S_{2} \in \mathscr{D}^{\prime}(\alpha, \infty)$. In fact, if $\psi$ is a function in $\mathscr{D}$ such that $\psi=1$ on $[-\alpha, \alpha]$, we can define $S_{1}=\psi S$ and $S_{2}=S-S_{1}$.

By the hypothesis, there exists $E \in \mathscr{D}^{\prime}(0, \infty)$ such that $S * E=\delta$. Hence

$$
S_{1} * E=\delta-S_{2} * E
$$

and $S_{2} * E \in \mathscr{D}^{\prime}(\alpha, \infty)$, by (3).

Consider now a solution $u_{1} \in \mathscr{D}^{\prime}(0, \infty)$ of the equation $S * u=S_{2} * E$. By assumption, $u_{1}$ exists. Also, since $S_{2} * E \in \mathscr{D}^{\prime}(\alpha, \infty)$ and $0 \in \operatorname{sing} \operatorname{supp} S, u_{1}$ is in $\mathscr{D}^{\prime}(\alpha, \infty)$ by Titchmarsh's theorem. It follows that

$$
S_{1} * u_{1}=S_{2} * E-S_{2} * u_{1}
$$

and $S_{2} * u_{1} \in \mathscr{D}^{\prime}(2 \alpha, \infty)$ by (3).

Suppose that $u_{k} \in \mathscr{D}^{\prime}(k \alpha, \infty)$ has been defined for $k \geqslant 1$. We then define $u_{k+1}$ as a solution in $\mathscr{D}^{\prime}(0, \infty)$ of the equation $S * u=S_{2} * u_{k}$. Since $S_{2} * u_{k} \in$ $\mathscr{D}^{\prime}((k+1) \alpha, \infty), u_{k+1}$ must be in $\mathscr{D}^{\prime}((k+1) \alpha, \infty)$ by Titchmarsh's theorem, and we have

$$
S_{1} * u_{k+1}=S_{2} * u_{k}-S_{2} * u_{k+1} \text {. }
$$

In this way we have defined a sequence of distributions $u_{k} \in \mathscr{D}^{\prime}(k \alpha, \infty)$ satisfying equations (16) and (17). Adding both sides of equations (15), (16), and (17) for $k=1,2, \ldots, n$, we obtain

$$
S_{1} *\left(E+\sum_{k=1}^{n+1} u_{k}\right)=\delta-S_{2} * u_{n+1}
$$

Since $u_{n} \in \mathscr{D}^{\prime}(n \alpha, \infty)$, the series $\sum_{k=1}^{\infty} u_{k}$ converges and $S_{2} * u_{n+1} \rightarrow 0$ in $\mathscr{D}^{\prime}(0, \infty)$, as $n \rightarrow \infty$. Thus, from (18) it follows that

$$
S_{1} *\left(E+\sum_{k=1}^{\infty} u_{k}\right)=\delta,
$$

i.e. $E_{1}=E+\sum_{k=1}^{\infty} u_{k}$ is a fundamental solution for $S_{1}$ in $\mathscr{D}^{\prime}(0, \infty)$.

The converse of Theorem 3 is also true.

THEOREM 4. Let $S$ be a distribution in $\mathscr{D}^{\prime}(0, \infty)$ and let $\alpha>0$. If $S$ admits a decomposition (14), where $S_{1} \in \mathscr{E}^{\prime}, S_{2} \in \mathscr{D}^{\prime}(\alpha, \infty)$, and $S_{1}$ satisfies the equivalent conditions of Corollary 1 , then $S * \mathscr{D}^{\prime}(0, \infty)=\mathscr{D}^{\prime}(0, \infty)$.

Proof. It suffices to construct a fundamental solution $E \in \mathscr{D}^{\prime}(0, \infty)$ for $S$.

By assumption, there exists $E_{1} \in \mathscr{D}^{\prime}(0, \infty)$ such that $S_{1} * E_{1}=\delta$. We now consider a solution $v_{1} \in \mathscr{D}^{\prime}(0, \infty)$ of the equation $S_{1} * v=-S_{2} * E_{1}$. Since $S_{2}$ is in 
$\mathscr{D}^{\prime}(\alpha, \infty), S_{2} * E_{1}$ is also in $\mathscr{D}^{\prime}(\alpha, \infty)$ by (3). But $0 \in \operatorname{sing} \operatorname{supp} S_{1}$, and so $v_{1} \in$ $\mathscr{D}^{\prime}(\alpha, \infty)$ by Titchmarsh's theorem. We also have

$$
S *\left(E_{1}+v_{1}\right)=\delta+S_{2} * v_{1} \text {. }
$$

Suppose that $v_{k}$ is defined for $k=1,2, \ldots, n$, so that $v_{k} \in \mathscr{D}^{\prime}(k \alpha, \infty)$ and

$$
S *\left(E_{1}+\sum_{k=1}^{n} v_{k}\right)=\delta+S_{2} * v_{n} \text {. }
$$

We define $v_{n+1}$ to be a solution in $\mathscr{D}^{\prime}(0, \infty)$ of the equation $S_{1} * v=-S_{2} * v_{n}$. By the same argument as above, we can show that $v_{n+1} \in \mathscr{D}^{\prime}((n+1) \alpha, \infty)$ and

$$
S *\left(E_{1}+\sum_{k=1}^{n+1} v_{k}\right)=\delta+S_{2} * v_{n+1} \text {. }
$$

We have thus constructed a sequence of distributions $v_{n} \in \mathscr{D}^{\prime}(n \alpha, \infty)$ which satisfy (19). But $v_{n} \rightarrow 0$, and therefore $S_{2} * v_{n} \rightarrow 0$ as $n \rightarrow \infty$, and the series $\sum_{n=1}^{\infty} v_{n}$ converges in $\mathscr{D}^{\prime}(0, \infty)$. Consequently, it follows from (19) that $E=E_{1}+\sum_{n=1}^{\infty} v_{n}$ is a fundamental solution for $S$ in $\mathscr{D}^{\prime}(0, \infty)$.

COROLlaRY 2. If $S \in \mathscr{D}^{\prime}(0, \infty)$, the following conditions are equivalent.

$\left(\mathrm{k}_{1}\right) S * \mathscr{D}^{\prime}(0, \infty)=\mathscr{D}^{\prime}(0, \infty)$.

$\left(\mathrm{k}_{2}\right)$ For some $\alpha>0$ (or equivalently, for every $\alpha>0$ ), $S$ admits a decomposition (14), where $S_{1} \in \mathscr{E}^{\prime}, S_{2} \in \mathscr{D}^{\prime}(\alpha, \infty)$, and $S_{1}$ satisfies the equivalent conditions in Corollary 1.

We now establish necessary and sufficient conditions on a distribution $S \in$ $\mathscr{S}^{\prime}(0, \infty)$ in order that $S * \mathscr{S}^{\prime}(0, \infty)=\mathscr{S}^{\prime}(0, \infty)$.

TheOREM 5. Let $S \in \mathscr{S}^{\prime}(0, \infty)$ and let $\hat{s}$ be the analytic continuation in $C_{-}$of the Fourier transform $\hat{S}$ of $S$. Then the following conditions are equivalent.

$\left(1_{1}\right) S * \mathscr{S}^{\prime}(0, \infty)=\mathscr{S}^{\prime}(0, \infty)$.

$\left(1_{2}\right) \hat{s}(\zeta) \neq 0$ for all $\zeta \in C_{-}$, and there exist positive integers $m, n$ and a constant $c>0$ such that

$$
|\hat{s}(\zeta)| \geqslant c(1+|\zeta|)^{-m} M_{-n}(\eta),
$$

where $\zeta=\xi+i \eta$ and $M_{n}$ is the function defined by (4).

Proof. It follows from $\left(\mathrm{l}_{1}\right)$ that there is $E \in \mathscr{S}^{\prime}(0, \infty)$ such that

$$
S * E=\delta \text {. }
$$

If $\hat{e}$ is the analytic continuation in $C_{-}$of the Fourier transform $\hat{E}$ of $E$, then (21) implies that $\hat{s}(\zeta) \hat{e}(\zeta)=1, \zeta \in C_{-}$. Hence $\hat{s}(\zeta) \neq 0$ for all $\zeta \in C_{-}$, and there are positive integers $m, n$ and a constant $C$ such that

$$
|1 / \hat{s}(\zeta)|=|\hat{e}(\zeta)| \leqslant C(1+|\zeta|)^{m} M_{n}(\eta),
$$

in view of (5). This is equivalent to (20) with $c=1 / C$.

Conversely, if the analytic continuation $\hat{S}$ of $\hat{S}$ satisfies conditions $\left(1_{2}\right)$, then $\hat{e}(\zeta)=1 / \hat{s}(\zeta)$ satisfies the estimate $(5)$ in $C_{-}$, and so $\hat{e}(\zeta)$ is the analytic continuation of the Fourier transform $\hat{E}$ of a distribution $E \in \mathscr{S}^{\prime}(0, \infty)$. Moreover, since $\hat{s}(\zeta) \hat{e}(\zeta)=1$, we have $S * E=\delta$, i.e. $E$ is a fundamental solution in $\mathscr{S}^{\prime}(0, \infty)$ for $S$. 


\section{REFERENCES}

1. L. Ehrenpreis, Solutions of some problems of division. IV, Amer. J. Math. 82 (1960), 522-588.

2. L. Hörmander, On the range of convolution operators, Ann. of Math. (2) 76 (1962), 148-170.

3. J. Mikusinski, Un simple demonstration du théorème de Titchmarsh sur la convolution, Bull. Acad. Polon. Sci. 7 (1959), 715 - 718.

4. L. Schwartz, Theorie des distributions. II, Hermann, Paris, 1951.

5. R. Shambayati and Z. Zielezny, On Fourier transforms of distributions with one-sided bounded support, Proc. Amer. Math. Soc. 88 (1983), 237-243.

6. E. C. Titchmarsh, Introduction to the theory of Fourier integrals, Clarendon Press, Oxford, 1937.

Department of Mathematics and Computer Science, Daemen College, BoX 686, AmHerst, New YORK 14226

Department of Mathematics, State University of New York at Buffalo, Buffalo, New York 14214 